\title{
Focal Points of the Preanesthesia Evaluation for Electroconvulsive Therapy in Patients with Depression: A Retrospective Analysis of Clinical Characteristics in Nonremission
}

\section{Lei Zou}

First Affiliated Hospital of Chongqing Medical University

\section{Xiao Li}

First Affiliated Hospital of Chongqing Medical University

\section{Qibin Chen}

First Affiliated Hospital of Chongqing Medical University

\section{Feng LV}

First Affiliated Hospital of Chongqing Medical University

Su Min ( $\sim$ ms89011068@163.com)

First Affiliated Hospital of Chongqing Medical University

\section{Research Article}

Keywords: ECT, retrospective analysis, nonremission, depression

Posted Date: January 19th, 2022

DOI: https://doi.org/10.21203/rs.3.rs-1220765/v1

License: (c) (i) This work is licensed under a Creative Commons Attribution 4.0 International License. Read Full License 


\section{Abstract}

Background: This study explored the patient clinical characteristics that may affect electroconvulsive therapy (ECT) efficacy, to enable improved focus during the evaluation and preparation before ECT.

Methods: Patients enrolling for ECT at the Department of Psychiatry and Anesthesiology of the First Affiliated Hospital of Chongqing Medical University from December 2017 to January 2019.Univariate and multivariate analyses were performed to identify the risk factors for nonremission. Variables with statistical significance in the univariate analysis were included in the multivariate logistic regression analysis to determine independent risk factors.

Results: In total, 874 cases showed efficacy in this study. After ECT treatment, 255 cases (29.2\%) exhibited nonremission. Multivariate logistic regression analysis of variables was performed, and the results showed that atherosclerosis (OR 8.072, 95\% $\mathrm{Cl} 2.442$ to $16.675 ; \mathrm{p}=0.001$ ), COPD (OR 2.919, 95\% $\mathrm{Cl} 1.240$ to $6.871 ; p=0.014$ ), diabetes (OR $2.202,95 \% \mathrm{Cl} 1.115$ to $4.348 ; p=0.023$ ) and smoking (OR $1.519,95 \% \mathrm{Cl} 1.015$ to $2.273 ; \mathrm{p}=0.042$ ) were independent risk factors for nonremission.

Conclusion: In the retrospective analysis, we found that atherosclerosis, diabetes, COPD or smoking may be high-risk factors for nonremission.

\section{Introduction}

Depression is a mood disorder characterized by persistent feelings of loss of interest along with a cluster of clinical symptoms. The World Health Organization projects that worldwide, depression will be the leading cause of disease burden by 2030 , and depression is a significant public health concern affecting 350 million people worldwide ${ }^{1,2}$. Despite the development of newer brain stimulation techniques and novel pharmacological agents, no treatment has approached the efficacy of electroconvulsive therapy (ECT) for depression, especially for patients whose medical or psychiatric condition requires a rapid and/or definite response ${ }^{3}$, and researchers have been trying to identify other methods to improve the efficacy of ECT.

Anesthesia is an indispensable part of ECT treatment, as it not only eliminates the patients' fear of the procedure, thereby reducing the stress response, but also reduces the incidence of adverse events, such as fracture, tooth injury, and cardiovascular and cerebrovascular accidents. Furthermore, the rational use of anesthetics such as propofol and ketamine can improve the efficacy of ECT ${ }^{4-6}$ and may confer at least a short-term advantage in terms of improving depressive symptoms at the early stages of ECT ${ }^{7}$. A series of studies conducted by our research group also supported these views ${ }^{8,9}$.

We studied the effects of ketamine in different age groups and confirmed its validity and safety. At the same time, by referring to the literature, we found that ECT was particularly effective in elderly individuals with depression ${ }^{10-12}$. However, there are differing views. Some studies have suggested that response and remission rates to pharmacotherapy and ECT are not sufficiently different between old-age and middle- 
age depression to be clinically significant ${ }^{13}$ or that effectiveness is independent of age ${ }^{14}$. In addition, elderly individuals may have more complications, such as hypertension, diabetes, atherosclerosis, and hyperlipidemia, which may aggravate the depressive symptoms of the patients ${ }^{15-17}$.

Combined with our research and current reports, we believe that patient age, medical complications, adjuvant anesthesia and other factors will affect the treatment of ECT and may be the reasons why depression is not alleviated after treatment with ECT. However, there is no comprehensive analysis of these factors. Therefore, the purpose of this study was to explore the risk factors that may affect the efficacy of ECT so that the anesthesia can be adjusted accordingly.

\section{Methods}

This study retrospectively included patients who enrolled for ECT at the Department of Psychiatry and Anesthesiology of the First Affiliated Hospital of Chongqing Medical University from December 2017 to January 2019. The study protocol was approved by the ethics committee of the hospital, and all aspects of the study complied with the Declaration of Helsinki. There is no need to obtain informed consent from patients since this is a retrospective study, and all the data were collected and analyzed anonymously.

The inclusion criteria were complete medical records, and subjects diagnosed with depression (Diagnostic and Statistical Manual of Mental Disorders, Fourth Edition) were included. We excluded patients diagnosed with other psychiatric diseases. We collected clinical data of patients through the electronic medical record system. Basic information on the patients, such as age, sex, body mass index (BMI), smoking history, drinking history, course of disease, marital status, and recurrent depression, was collected. Education level was divided into illiterate, nine-year compulsory education and high school, and above. Smoking status was classified as nonsmoking and smoking. Additionally, participants were divided into a nondrinking group and a drinking group. Marital status was classified as unmarried, married, divorced and widowed. According to age, participants were classified as minor ( $<18$ years), youth (18-44), middle age (45-59) and old age ( $\geq 60$ years). The physical examination included measurements of height and weight. We measured height and weight to the nearest $1 \mathrm{~cm}$ and $0.1 \mathrm{~kg}$, respectively. BMI was calculated as the weight in kilograms divided by the height in meters squared. Individuals with BMIs $\geq 24 \mathrm{~kg} / \mathrm{m}^{2}$ were considered to be overweight, and those with BMls $\geq 28 \mathrm{~kg} / \mathrm{m}^{2}$ were considered to be obese. Complications included diabetes, hypertension, atherosclerosis, hyperlipidemia, chronic obstructive pulmonary disease (COPD), and hypothyroidism. Outpatients were excluded because of incomplete information.

Depressive symptoms were assessed using the 24-item Hamilton Depression Rating Scale (HAMD-24) at baseline and after the end of the ECT session. Response was defined as a $\geq 50 \%$ decrease in HAMD-24 scores from baseline. Remission was defined as a HAMD-24 score $\leq 10$ after the last two consecutive ECT sessions. 
Statistical analyses were performed using IBM SPSS Statistics 26.0 software (IBM Corporation, Armonk, NY). Univariate and multivariate analyses were performed to identify the risk factors for nonremission. The Shapiro-Wilk test was used to determine whether the course of disease and number of treatments were normally distributed. All measurement data are presented as the mean \pm SD (standard deviation) when data met the criteria for normality with $p>0.10$. Otherwise, the data are presented as the median (interquartile range, IQR). When the data met the criteria for normality and homogeneity of variance, statistical analysis between groups was performed using independent samples t-tests. Otherwise, measurement data with a nonnormal distribution were analyzed by means of the Mann-Whitney $U$ test. Variables with statistical significance in the univariate analysis were included in the multivariate logistic regression analysis to determine independent risk factors. $p$ values $<0.05$ were considered statistically significant.

\section{Results}

During this period, 1617 patients received ECT, of which 903 were diagnosed with depression. Because not all the information could be obtained for outpatients, 874 inpatients were included. According to the diagnostic criteria, 619 patients achieved remission, with a remission rate of $70.8 \%$. A total of 255 people did not reach the remission standard, accounting for $29.2 \%$ of the patients (Fig. 1, Table 1).

Table 1

General condition of patients with

\begin{tabular}{|lll|}
\hline \multicolumn{3}{|c|}{ depression after ECT [ cases (\%) ] } \\
\hline Total & remission & nonremission \\
\hline $874(100)$ & $619(70.8)$ & $255(29.2)$ \\
\hline
\end{tabular}

None of the patients had severe complications. Their clinical features are shown in Table 2, and the results of the univariate analyses are also included. The results showed that there were no significant differences in remission rates among participants according to sex $(P=0.774)$, education $(P=0.304)$, alcohol use $(P=0.199)$ and family history of depression $(P=0.687)$. There were significant differences in age $(P=0.003)$, hypertension $(P=0.002)$, diabetes $(P=0.000)$, marital status $(P=0.018), B M I(P=0.018)$, smoking $(P=0.014)$, atherosclerosis $(P=0.000)$, COPD $(P=0.005)$, hyperlipidemia $(P=0.000)$, hypothyroidism ( $P=0.038)$, history of anesthesia $(P=0.014)$, first-onset depression $(P=0.004)$, course of depression $(P=0.000)$, and number of ECTs $(P=0.000)$ (Table 2, Table 3$)$.

Table 2. Univariate analyses of the risk factors for nonremission 


\begin{tabular}{|c|c|c|c|c|c|c|}
\hline & \multicolumn{2}{|c|}{ remission $(n=619)$} & \multicolumn{2}{|c|}{ nonremission $(\mathrm{n}=255)$} & \multirow{2}{*}{$\begin{array}{l}x^{2} \\
14.093\end{array}$} & \multirow{2}{*}{$\begin{array}{l}p \\
0.003^{*}\end{array}$} \\
\hline Age(year) & & & & & & \\
\hline$<18$ & 111 & $17.9 \%$ & 29 & $11.4 \%$ & & \\
\hline $18-44$ & 289 & $46.7 \%$ & 109 & $42.7 \%$ & & \\
\hline $45-59$ & 145 & $23.4 \%$ & 66 & $25.9 \%$ & & \\
\hline$\geq 60$ & 74 & $12.0 \%$ & 51 & $20.0 \%$ & & \\
\hline Gender & & & & & 0.083 & 0.774 \\
\hline male & 217 & $35.1 \%$ & 92 & $36.1 \%$ & & \\
\hline famale & 402 & $64.9 \%$ & 163 & $63.9 \%$ & & \\
\hline Marital status & & & & & 10.318 & $0.018^{*}$ \\
\hline unmarried & 257 & $41.5 \%$ & 79 & $31.0 \%$ & & \\
\hline married & 321 & $51.9 \%$ & 150 & $58.8 \%$ & & \\
\hline divorced & 28 & $4.5 \%$ & 19 & $7.5 \%$ & & \\
\hline widowed & 13 & $2.1 \%$ & 7 & $2.7 \%$ & & \\
\hline Education & & & & & 2.379 & 0.304 \\
\hline illiterate & 29 & $4.7 \%$ & 16 & $6.3 \%$ & & \\
\hline 9-years compulsory education & 321 & $51.9 \%$ & 119 & $46.7 \%$ & & \\
\hline high school or above & 269 & $43.5 \%$ & 120 & $47.1 \%$ & & \\
\hline BMI & & & & & 8.030 & $0.018 *$ \\
\hline$<18.5$ & 20 & $3.2 \%$ & 5 & $2.0 \%$ & & \\
\hline $18.5-23.9$ & 565 & $91.3 \%$ & 223 & $87.5 \%$ & & \\
\hline$\geq 24$ & 34 & $5.5 \%$ & 27 & $10.6 \%$ & & \\
\hline Smoking & & & & & 6.100 & $0.014^{*}$ \\
\hline yes & 89 & $14.4 \%$ & 54 & $21.2 \%$ & & \\
\hline no & 530 & $85.6 \%$ & 201 & $78.8 \%$ & & \\
\hline Alcohol use & & & & & 1.647 & 0.199 \\
\hline yes & 88 & $14.2 \%$ & 45 & $17.6 \%$ & & \\
\hline no & 531 & $85.8 \%$ & 210 & $82.4 \%$ & & \\
\hline Diabetes & & & & & 13.863 & $0.000 *$ \\
\hline
\end{tabular}




\begin{tabular}{|c|c|c|c|c|c|c|}
\hline yes & 22 & $3.6 \%$ & 25 & $9.8 \%$ & & \\
\hline no & 597 & $96.4 \%$ & 230 & $90.2 \%$ & & \\
\hline Hypertension & & & & & 9.169 & $0.002^{*}$ \\
\hline yes & 43 & $6.9 \%$ & 34 & $13.3 \%$ & & \\
\hline no & 576 & $93.1 \%$ & 221 & $86.7 \%$ & & \\
\hline Atherosclerosis & & & & & 25.586 & $0.000 *$ \\
\hline yes & 4 & $0.6 \%$ & 16 & $6.3 \%$ & & \\
\hline no & 615 & $99.4 \%$ & 239 & $93.7 \%$ & & \\
\hline Hyperlipidemia & & & & & 13.416 & $0.000 *$ \\
\hline yes & 27 & $4.4 \%$ & 28 & $11.0 \%$ & & \\
\hline no & 592 & $95.6 \%$ & 227 & $89.0 \%$ & & \\
\hline COPD & & & & & 7.893 & $0.005^{\star}$ \\
\hline yes & 12 & $1.9 \%$ & 14 & $5.5 \%$ & & \\
\hline no & 607 & $98.1 \%$ & 241 & $94.5 \%$ & & \\
\hline Hypothyroidism & & & & & 4.301 & $0.038 *$ \\
\hline yes & 27 & $4.4 \%$ & 20 & $7.8 \%$ & & \\
\hline no & 592 & $95.6 \%$ & 235 & $92.2 \%$ & & \\
\hline History of anesthesia & & & & & 5.979 & $0.014 *$ \\
\hline yes & 156 & $25.2 \%$ & 85 & $33.3 \%$ & & \\
\hline no & 463 & $74.8 \%$ & 170 & $66.7 \%$ & & \\
\hline Family history of depression & & & & & 0.163 & 0.687 \\
\hline yes & 94 & $15.2 \%$ & 36 & $14.1 \%$ & & \\
\hline no & 525 & $84.8 \%$ & 219 & $85.9 \%$ & & \\
\hline First-onset depression & & & & & 8.231 & $0.004^{*}$ \\
\hline yes & 238 & $38.4 \%$ & 72 & $28.2 \%$ & & \\
\hline no & 381 & $61.6 \%$ & 183 & $71.8 \%$ & & \\
\hline
\end{tabular}

Table 3. Univariate analyses of the risk factors for nonremission (continued) 


\begin{tabular}{|lllll|}
\hline & remission $(\mathrm{n}=619)$ & nonremission $(\mathrm{n}=255)$ & $Z$ & $P$ \\
\hline Course of depression(month) & $15(5,48)$ & $36(8,75)$ & -24.099 & $0.000^{*}$ \\
\hline Number of ECT & $10(8,12)$ & $9(7,12)$ & -28.031 & $0.000^{*}$ \\
\hline
\end{tabular}

Multivariate logistic regression analysis of variables with $\mathrm{P}<0.05$ was performed, and the results showed that atherosclerosis, COPD, diabetes and smoking were independent risk factors for nonremission. It can be seen from the data that the number of patients with these clinical characteristics who had nonremission increased by 8.072-, 2.919-, 2.202- and 1.519-fold, respectively (Table 4, Fig. 2).

Table 4. Multivariate analyses of the risk factors for nonremission 


\begin{tabular}{|c|c|c|c|}
\hline & $B$ & OR $(95 \% \mathrm{Cl})$ & $P$ \\
\hline \multicolumn{4}{|l|}{ Age } \\
\hline minors & & 1.000 (reference) & 0.778 \\
\hline youth & 0.145 & $1.156(0.675-1.979)$ & 0.597 \\
\hline middle age & 0.105 & $1.111(0.562-2.198)$ & 0.762 \\
\hline old age & -0.119 & $0.888(0.398-1.978)$ & 0.771 \\
\hline \multicolumn{4}{|c|}{ Marital status } \\
\hline unmarried & & 1.000 (reference) & 0.629 \\
\hline married & -0.096 & $0.909(0.575-1.436)$ & 0.682 \\
\hline divorced & 0.343 & $1.409(0.685-2.900)$ & 0.352 \\
\hline widowed & -0.012 & $0.988(0.318-3.070)$ & 0.983 \\
\hline \multicolumn{4}{|l|}{ BMI } \\
\hline$<18.5$ & & 1.000 (reference) & 0.273 \\
\hline $18.5-23.9$ & 0.462 & $1.588(0.559-4.510)$ & 0.385 \\
\hline$\geq 24$ & 0.859 & $2.361(0.728-7.660)$ & 0.152 \\
\hline \multicolumn{4}{|l|}{ Smoking } \\
\hline \multicolumn{4}{|l|}{ no } \\
\hline yes & 0.418 & $1.519(1.015-2.273)$ & $0.042^{\star}$ \\
\hline \multicolumn{4}{|l|}{ Diabetes } \\
\hline \multicolumn{4}{|l|}{ no } \\
\hline yes & 0.789 & $2.202(1.115-4.348)$ & $0.023^{*}$ \\
\hline \multicolumn{4}{|c|}{ Hypertension } \\
\hline \multicolumn{4}{|l|}{ no } \\
\hline yes & 0.167 & $1.181(0.652-2.140)$ & 0.583 \\
\hline \multicolumn{4}{|c|}{ Atherosclerosis } \\
\hline \multicolumn{4}{|l|}{ no } \\
\hline yes & 2.088 & $8.072(2.442-16.675)$ & $0.001^{*}$ \\
\hline \multicolumn{4}{|c|}{ Hyperlipidemia } \\
\hline no & & & \\
\hline
\end{tabular}




\begin{tabular}{|llll|}
\hline yes & 0.627 & $1.872(0.991-3.539)$ & 0.053 \\
\hline no & & & \\
yes & 1.071 & $2.919(1.240-6.871)$ & $0.014^{*}$ \\
\hline Hypothyroidism & & & \\
\hline no & & & \\
\hline yes & 0.496 & $1.643(0.865-3.121)$ & 0.129 \\
\hline History of anesthesia & & & \\
\hline no & & & \\
\hline yes & 0.346 & $1.414(0.992-2.016)$ & 0.055 \\
\hline First-onset depression & & & \\
\hline no & & & \\
\hline yes & 0.257 & $1.293(0.912-1.833)$ & 0.148 \\
\hline Course of depression & 0.002 & $1.002(1.000-1.004)$ & 0.075 \\
\hline Number of ECT & -0.127 & $0.881(0.834-0.930)$ & $0.000 *$ \\
\hline
\end{tabular}

\section{Discussion}

At present, research on improving the efficacy of ECT is carried out mainly by psychiatrists in terms of aspects such as electroshock mode, electric quantity and stimulation site ${ }^{18}$, while anesthesiologists are concerned mainly with the different anesthetic compounds or administration modes ${ }^{19,20}$. By analyzing the clinical characteristics of nonremission patients, this study intended to improve the evaluation and regulation of patients before anesthesia to further improve the efficacy of ECT. We found that atherosclerosis, diabetes, COPD and smoking may be high-risk factors for nonremission ECT. To date, there is no relevant research, but it is certain that these factors will affect the symptoms of depression.

There is an interactive relationship between depression and atherosclerosis. Studies have shown that depression increases the risk of atherosclerosis. First, from the perspective of pathophysiology, depression is accompanied by immune dysregulation ${ }^{21}$, and in this context, the levels of a variety of peripheral inflammatory biomarkers are increased ${ }^{22}$. On the other hand, there are alterations in the nitric oxide (NO) system ${ }^{23}$. Endothelium-derived NO, through its vasodilator properties, participates in the modulation of vascular tone ${ }^{24}$. These factors promote atherosclerosis. In addition, the behavioral effects of depressive symptoms are considered to be another source of subclinical atherosclerosis, such as sleep disorders, sedentary behavior and obesity, smoking and alcohol consumption. Sleep disorders lead to 
impaired vascular endothelial function ${ }^{25}$, sedentary behavior causes intimal thickening of arteries ${ }^{26}$, and smoking and obesity lead to the formation of atherosclerotic plaques ${ }^{27}$, all of which contribute to an increased atherosclerotic burden. Depression and atherosclerosis have a common pathophysiological basis $^{28,29}$, and it has been confirmed that intracranial atherosclerosis will aggravate the symptoms of depression ${ }^{30}$. It has been reported that depression patients with atherosclerosis have a poor response to drug treatment ${ }^{31}$. However, ECT treatment has not been reported. Our study found that atherosclerosis is the most important reason for nonremission after ECT, showed that atherosclerosis may have an impact on depression in another way, and indicated that it will influence the treatment efficacy. Some studies have shown that the treatment of atherosclerosis can reduce depression ${ }^{32}$. Therefore, we should treat atherosclerosis when evaluating such patients, as this may improve the efficacy of ECT.

Evidence has also suggested a bidirectional relationship between diabetes and depression ${ }^{33,34}$. Numerous studies have confirmed that the course of depression in patients with diabetes is more severe and that depression episode relapses are more frequent. From a pathophysiological perspective, depression is highly consistent with diabetic complications. These complications include macrovascular complications (such as coronary artery disease), microvascular complications (diabetic retinopathy, neuropathy, nephropathy or end-stage renal disease) and bidirectional complications (depression may increase the risk of diabetic complications). On the other hand, these complications also affect the occurrence and development of depression ${ }^{17,35}$. Our research also shows that diabetes is a high-risk factor affecting the efficacy of ECT, which may be related to the aggravation of depressive symptoms by diabetic complications. The treatment of diabetes can reduce the symptoms of depression ${ }^{36}$, which may help to improve the efficacy of ECT. However, there is still a lack of prospective studies to support this conclusion.

Our study also found that COPD and smoking are key indicators affecting the efficacy of ECT. These two factors are different from the previous two high-risk factors. Although they do not directly cause vascular disease, they affect the development of depression in other ways. COPD causes hypoxia by destroying the lung capillary bed and causing poor airway ventilation, leading to a decrease in neurotransmitter serotonin activity and eventually depression ${ }^{37,38}$. Hypoxia may also cause global suppression of cerebral metabolism (energy production), leading to depression ${ }^{39,40}$. A series of symptoms of COPD are also causes of depression, and dyspnea, as the core symptom of COPD, may play an important role in the causal relationship between COPD and depression ${ }^{41,42}$. These patients are less active, have a lower quality of life and have a worse mood. Smoking has an impact on vascular disease and lung disease, aggravating these diseases ${ }^{43}, 44$. Smoking may also be an independent risk factor for depression ${ }^{45,46}$. This may be the reason why smoking affects the efficacy of ECT.

Treatment with these risk factors can improve the depressive symptoms of patients, but the correlation between these factors and ECT has not been reported. In addition, psychiatrists can improve the efficacy of ECT by changing the stimulation power, using different stimulation sites, and changing the stimulation 
mode, among other actions ${ }^{18,47,48}$. Therefore, we hope that when anesthesiologists evaluate patients, they will pay attention to and address these risk factors to further improve the efficacy of ECT.

\section{Limitations}

There were several limitations in this study. First, although these risk factors certainly have an effect on depression, we were unable to confirm their direct relationship with the risk of nonremission. Second, we could not rule out interactions between these factors. Third and most importantly, at present, there is a lack of prospective studies to confirm these findings. Therefore, in the next phase of the study, we will use the experience of enhanced postoperative recovery (ERAS) to treat depression patients who have these risk factors during the anesthesia evaluation.

\section{Declarations}

\section{Ethics approval and consent to participate}

The study protocol was approved by the Ethics Committee of the First Affiliated Hospital of Chongqing Medical University (No.20140409) and a waiver of informed consent was granted. The study was carried out in accordance with Helsinki declaration.

\section{Consent for publication}

Not Applicable.

\section{Competing interests}

Authors have no conflicts of interest to report.

\section{Funding}

This work was supported by the National Natural Science Foundation of China grant (81271501), as well as a grant from National Key Clinical Special Construction Project (No.2011-170).

\section{Author contributions}

Lei Zou made statistics and wrote the manuscript. Su Min designed the research experiments. Qibin Chen helped with data collection. Xiao Li helped with data collection and ECT assessment. Feng Lv helped with data collection and made statistics.

\section{Acknowledgments}

The authors thank Xiaoni Zhong and Jing Zhao, Professors of Chongqing Medical University, for their constructive comments during the review process. We also thank Yao Gan, Associate Professor in the 
Department of Psychiatry of the First Affiliated Hospital of Chongqing Medical University, for her helpful comments.

\section{Data availability statements}

The datasets generated during the current study are not publicly available due to involving patient privacy but are available from the corresponding author on reasonable request.

\section{References}

1. Ali SS, Khan SA, Khosa F et al. Noninvasive assessment of subclinical atherosclerosis in persons with symptoms of depression. Atherosclerosis. 2017; 264: 92-99.

2. Dwyer JB, Aftab A, Radhakrishnan R et al. Hormonal Treatments for Major Depressive Disorder: State of the Art. Am J Psychiatry. 2020; 177: 686-705.

3. Kellner $\mathrm{CH}$, Obbels J, Sienaert P. When to consider electroconvulsive therapy (ECT). Acta Psychiatr Scand. 2020; 141: 304-315.

4. Kellner CH, losifescu DV. Ketamine and ECT: better alone than together. Lancet Psychiatry. 2017; 4: 348-349.

5. Basso L, Bönke L, Aust $S$ et al. Antidepressant and neurocognitive effects of serial ketamine administration versus ECT in depressed patients. J Psychiatr Res. 2020; 123: 1-8.

6. Sartorius A, Beuschlein J, Remennik D et al. Empirical ratio of the combined use of S-ketamine and propofol in electroconvulsive therapy and its impact on seizure quality. Eur Arch Psychiatry Clin Neurosci. 2021; 271: 457-463.

7. Zheng W, Li XH, Zhu XM et al. Adjunctive ketamine and electroconvulsive therapy for major depressive disorder: A meta-analysis of randomized controlled trials. J Affect Disord. 2019; 250: 123-131.

8. Chen Q, Min S, Hao X et al. Effect of Low Dose of Ketamine on Learning Memory Function in Patients Undergoing Electroconvulsive Therapy-A Randomized, Double-Blind, Controlled Clinical Study. J ECT. 2017; 33: 89-95.

9. Zou L, Min S, Chen Q, Li X, Ren L. Subanesthetic dose of ketamine for the antidepressant effects and the associated cognitive impairments of electroconvulsive therapy in elderly patients-A randomized, double-blind, controlled clinical study. Brain Behav. 2021; 11: e01775.

10. van Diermen $L$, van den Ameele $S$, Kamperman AM et al. Prediction of electroconvulsive therapy response and remission in major depression: meta-analysis. Br J Psychiatry. 2018; 212: 71-80.

11. Güney P, Ekman CJ, Hammar $\AA$ et al. Electroconvulsive Therapy in Depression: Improvement in Quality of Life Depending on Age and Sex. J ECT. 2020; 36: 242-246.

12. Meyer JP, Swetter SK, Kellner CH. Electroconvulsive Therapy in Geriatric Psychiatry: A Selective Review. Clin Geriatr Med. 2020; 36: 265-279. 
13. Mitchell AJ, Subramaniam H. Prognosis of depression in old age compared to middle age: a systematic review of comparative studies. Am J Psychiatry. 2005; 162: 1588-1601.

14. Socci C, Medda P, Toni C et al. Electroconvulsive therapy and age: Age-related clinical features and effectiveness in treatment resistant major depressive episode. J Affect Disord. 2018; 227: 627-632.

15. Liguori I, Russo G, Curcio F et al. Depression and chronic heart failure in the elderly: an intriguing relationship. J Geriatr Cardiol. 2018; 15: 451-459.

16. Agustini B, Lotfaliany M, Woods RL et al. Patterns of Association between Depressive Symptoms and Chronic Medical Morbidities in Older Adults. J Am Geriatr Soc. 2020; 68: 1834-1841.

17. van Sloten TT, Sedaghat S, Carnethon MR, Launer LJ, Stehouwer C. Cerebral microvascular complications of type 2 diabetes: stroke, cognitive dysfunction, and depression. Lancet Diabetes Endocrinol. 2020; 8: 325-336.

18. Semkovska M, Landau S, Dunne R et al. Bitemporal Versus High-Dose Unilateral Twice-Weekly Electroconvulsive Therapy for Depression (EFFECT-Dep): A Pragmatic, Randomized, Non-Inferiority Trial. Am J Psychiatry. 2016; 173: 408-417.

19. Ren L, Deng J, Min S, Peng L, Chen Q. Ketamine in electroconvulsive therapy for depressive disorder: A systematic review and meta-analysis. J Psychiatr Res. 2018; 104: 144-156.

20. Dong J, Min S, Qiu H, Chen Q, Ren L. Intermittent administration of low dose ketamine can shorten the course of electroconvulsive therapy for depression and reduce complications: A randomized controlled trial. Psychiatry Res. 2019; 281: 112573.

21. Chrysohoou C, Kollia N, Tousoulis D. The link between depression and atherosclerosis through the pathways of inflammation and endothelium dysfunction. Maturitas. 2018; 109: 1-5.

22. Osimo EF, Pillinger T, Rodriguez IM, Khandaker GM, Pariante CM, Howes OD. Inflammatory markers in depression: A meta-analysis of mean differences and variability in 5,166 patients and 5,083 controls. Brain Behav Immun. 2020; 87: 901-909.

23. Chrapko WE, Jurasz P, Radomski MW, Lara N, Archer SL, Le Mellédo JM. Decreased platelet nitric oxide synthase activity and plasma nitric oxide metabolites in major depressive disorder. Biol Psychiatry. 2004; 56: 129-134.

24. Hess S, Baker G, Gyenes G, Tsuyuki R, Newman S, Le Melledo JM. Decreased serum L-arginine and Lcitrulline levels in major depression. Psychopharmacology (Berl). 2017; 234: 3241-3247.

25. Del Brutto OH, Mera RM, Zambrano M, Simon LV, Matcha GV, Castillo PR. Sleep quality correlates with the carotid intima-media thickness in stroke-free community-dwelling adults living in rural Ecuador. The Atahualpa Project. Sleep Med. 2019; 55: 22-25.

26. Huang Y, Li L, Gan Y et al. Sedentary behaviors and risk of depression: a meta-analysis of prospective studies. Trans/ Psychiatry. 2020; 10: 26.

27. Ghosh RK, Ball S, Prasad V, Gupta A. Depression in heart failure: Intricate relationship, pathophysiology and most updated evidence of interventions from recent clinical studies. Int $J$ Cardiol. 2016; 224: 170-177. 
28. Saleptsis VG, Labropoulos N, Halaris A, Angelopoulos NV, Giannoukas AD. Depression and atherosclerosis. Int Angiol. 2011; 30: 97-104.

29. Pizzi C, Santarella L, Costa MG et al. Pathophysiological mechanisms linking depression and atherosclerosis: an overview. J Biol Regul Homeost Agents. 2012; 26: 775-782.

30. Chen YK, Qu JF, Xiao WM et al. Intracranial Atherosclerosis and Poststroke Depression in Chinese Patients with Ischemic Stroke. J Stroke Cerebrovasc Dis. 2016; 25: 998-1004.

31. Paranthaman R, Greenstein A, Burns AS, Heagerty AM, Malik RA, Baldwin RC. Relationship of endothelial function and atherosclerosis to treatment response in late-life depression. Int $J$ Geriatr Psychiatry. 2012; 27: 967-973.

32. Li L, Yu AL, Wang ZL et al. Chaihu-Shugan-San and absorbed meranzin hydrate induce antiatherosclerosis and behavioral improvements in high-fat diet $\mathrm{ApoE}(-/-)$ mice via anti-inflammatory and BDNF-TrkB pathway. Biomed Pharmacother. 2019; 115: 108893.

33. de Groot M, Anderson R, Freedland KE, Clouse RE, Lustman PJ. Association of depression and diabetes complications: a meta-analysis. Psychosom Med. 2001; 63: 619-630.

34. Chen PC, Chan YT, Chen HF, Ko MC, Li CY. Population-based cohort analyses of the bidirectional relationship between type 2 diabetes and depression. Diabetes Care. 2013; 36: 376-382.

35. Nouwen A, Adriaanse MC, van Dam K et al. Longitudinal associations between depression and diabetes complications: a systematic review and meta-analysis. Diabet Med. 2019; 36: 1562-1572.

36. Kessing LV, Rytgaard HC, Ekstrøm CT, Knop FK, Berk M, Gerds TA. Antidiabetes Agents and Incident Depression: A Nationwide Population-Based Study. Diabetes Care. 2020; 43: 3050-3060.

37. Kent BD, Mitchell PD, McNicholas WT. Hypoxemia in patients with COPD: cause, effects, and disease progression. Int J Chron Obstruct Pulmon Dis. 2011; 6: 199-208.

38. Riblet NB, Gottlieb DJ, Hoyt JE, Watts BV, Shiner B. An analysis of the relationship between chronic obstructive pulmonary disease, smoking and depression in an integrated healthcare system. Gen Hosp Psychiatry. 2020; 64: 72-79.

39. Bogdanova OV, Abdullah O, Kanekar S, Bogdanov VB, Prescot AP, Renshaw PF. Neurochemical alterations in frontal cortex of the rat after one week of hypobaric hypoxia. Behav Brain Res. 2014; 263: 203-209.

40. Kious BM, Kondo DG, Renshaw PF. Living High and Feeling Low: Altitude, Suicide, and Depression. Harv Rev Psychiatry. 2018; 26: 43-56.

41. Pollok J, van Agteren JE, Carson-Chahhoud KV. Pharmacological interventions for the treatment of depression in chronic obstructive pulmonary disease. Cochrane Database Syst Rev. 2018; 12 : CD012346.

42. Schuler M, Wittmann M, Faller H, Schultz K. The interrelations among aspects of dyspnea and symptoms of depression in COPD patients - a network analysis. J Affect Disord. 2018; 240: 33-40.

43. Ho C, Tan E, Ho R, Chiu M. Relationship of Anxiety and Depression with Respiratory Symptoms: Comparison between Depressed and Non-Depressed Smokers in Singapore. Int J Environ Res Public 
Health. 2019; 16.

44. Parikh NS, Salehi Omran S, Kamel H, Elkind M, Willey J. Symptoms of depression and active smoking among survivors of stroke and myocardial infarction: An NHANES analysis. Prev Med. 2020; 137: 106131.

45. Fluharty M, Taylor AE, Grabski M, Munafò MR. The Association of Cigarette Smoking With Depression and Anxiety: A Systematic Review. Nicotine Tob Res. 2017; 19: 3-13.

46. Prochaska JJ, Das S, Young-Wolff KC. Smoking, Mental Illness, and Public Health. Annu Rev Public Health. 2017; 38: 165-185.

47. Rong H, Xu SX, Zeng J et al. Study protocol for a parallel-group, double-blinded, randomized, controlled, noninferiority trial: the effect and safety of hybrid electroconvulsive therapy (Hybrid-ECT) compared with routine electroconvulsive therapy in patients with depression. BMC Psychiatry. 2019; 19: 344

48. Elias A, Thomas N, Sackeim HA. Electroconvulsive Therapy in Mania: A Review of 80 Years of Clinical Experience. Am J Psychiatry. 2021; 178: 229-239.

\section{Figures}

\section{Figure 1}

Clinical research flow chart

\section{Figure 2}

Multivariate analyses of the risk factors for nonremission 